\section{Neuroplasticity in visual impairments}

\author{
Paulo Ramiler Silva, ${ }^{1}$ Tiago Farias, ${ }^{1}$ \\ Fernando Cascio, ${ }^{1}$ Levi dos Santos, ${ }^{1}$ \\ Vinícius Peixoto, ${ }^{1}$ Eric Crespo, ${ }^{1}$ \\ Carla Ayres, ${ }^{1}$ Marcos Ayres, 1,2 \\ Victor Marinho, 1,2 \\ Victor Hugo Bastos, ${ }^{2,3}$ Pedro Ribeiro, ${ }^{4}$ \\ Bruna Velasques, 4 Marco Orsini, 5-7 \\ Rossano Fiorelli, ${ }^{6}$ \\ Marcos R.G. de Freitas, ${ }^{8}$ \\ Silmar Teixeira ${ }^{1,2}$
}

1Neuro-innovation Technology \& Brain

Mapping Laboratory, Federal

University of Piauí, Parnaíba;

2The Northeast Biotechnology Network, Federal University of Piauí, Teresina;

${ }^{3}$ Brain Mapping and Functionality

Laboratory, Federal University of Piauí,

Parnaíba; ${ }^{4}$ Brain Mapping and Sensory-

Motor Integration Laboratory, Federal

University of Rio de Janeiro, Rio de

Janeiro; 5Master's Program in Local

Development Program, University

Center Augusto Motta - UNISUAM, Rio

de Janeiro; 'Master's Program in

Health Sciences Applied - Vassouras

University, Rio de Janeiro;

7CASF- Ramon Pereira de Freitas -

Department of Neurology; 8Department of Neurology, Federal University of Rio de Janeiro, Rio de Janeiro, Brazil.

\footnotetext{
Abstract

The visual acuity loss enables the brain to access new pathways in the quest to overcome the visual limitation and this is wellknown as neuroplasticity which have mechanisms to cortical reorganization. In this review, we related the evidences about the neuroplasticity as well as cortical anatomical differences and functional repercussions in visual impairments. We performed a systematic review of PUBMED database, without date or status publication restrictions. The findings demonstrate that the visual impairment produce a compensatory sensorial effect, in which non-visual areas are related to both cross (visual congenital) and multimodal (late blind) neuroplasticity.
}

\section{Introduction}

\section{Research Highlights}

- The CNS is recognized as a constantly changing system. This characteristic called neuroplasticity consists of this tissue ability to reorganize as a consequence of normal development, during the learning process or after damage to the CNS. Some evidence that the sensory modality loss can trigger a reorganization of the CNS, especially in the cortex, expanding and making more functional the information processing provided by the intact senses. These neural changes can be explained through two theories: cross-plasticity and Multimodal plasticity.

- The occipital cortex activation for nonvisual tasks results in better performance in early and congenital visual impaired. Such improvement in the non-visual information processing is related to the elimination reduction process of non-functional synapses in the occipital cortex. This elimination process, dependent experience, enables the brain to test innumerable synapses in the occipital cortex and eliminate ineffective synapses for vision but for other functions may be useful.

- The brain plasticity occurs in individuals who lost sight in the first years of life both from the functional point of view and in the recruited brain area extension. The cross-plasticity occurs in response to vision sensory deprivation in visually impaired individuals. The multimodal plasticity refers to the balance alteration in the neural networks, due to the vision absence, which enables the activation of preexisting pathways that were being inhibited by vision.

- The cross-plasticity and multimodal theories corroborate for studies that have shown that visual cortical areas are recruited after visual loss for the processing of non-visual information. Cross-neuroplasticity has been associated with recruitment of visual brain areas during the sound and auditory processing. The hearing becomes more functional due it is frequently recruited to locate auditory stimuli, tactile and smell perception.

\section{Background}

It is estimated that by 2020 the popula-
Correspondence: Victor Marinho, Federal University of Piauí, Neuro-innovation Technology \& Brain Mapping Laboratory Av. São Sebastião nº2819 - Nossa Sra. de Fátima - Parnaíba, PI, CEP: 64202-020, Brazil. Tel.: +55 86 994178117. E-mail: victormarinhophb@hotmail.com

Key words: Neuroplasticity, vision, central nervous system, visual impairments.

Contributions: the authors contributed equally.

Conflict of interest: the authors declare no potential conflict of interest.

Funding: none.

Received for publication: 21 July 2017.

Revision received: 10 November 2017.

Accepted for publication: 25 December 2017.

This work is licensed under a Creative Commons Attribution NonCommercial 4.0 License (CC BY-NC 4.0).

CCopyright P.R. Silva et al., 2018

Licensee PAGEPress, Italy

Neurology International 2018; 10:7326

doi:10.4081/ni.2018.7326

tion of the visually impaired reaches 75 million people worldwide ${ }^{1}$, this fact shows the importance of understanding the neuronal changes that occur in these individuals, in order to enable the development of new treatment alternatives and understanding of how the brain behaves in the sense absence. However, science still cannot explain how neuroplasticity may become functional for all visual impaired (VI) and thus ensure that they can have the same adaptive enhancements. ${ }^{2,3}$

Brodmann, 4 in 1909, classified the human brain functionally in 52 areas, believing that science was finally able to explain the functioning of this organ. ${ }^{5}$ Hubel and Wiesel, 6 later to receive the Nobel Prize in medicine in a pioneering study, uncovered the primary visual cortex characteristics (area 17 of Brodmann). From experiments with monkeys, which they were subjected to radioactive amino acids in the right eye and a subsequent radiographic of the visual cortex analysis and its other cortical areas associated with vision. Nevertheless, the brain classification into areas was an attempt to simplify something extremely complex. To this day, the human brain remains an enigma for neuroscientists since the understanding of its functioning goes beyond the demarcation of brain areas associated with a given function. It is known that if a child undergoes a hemispherectomy, the surviving hemisphere may 
assume some the lost hemisphere functions. This contributes to strengthening the thinking that our brain is dynamic and capable of undergoing changes that allow for functional adaptations.5,7 Neuroplasticity experts call this cerebral dynamic behavior as a continuous alteration, either through the new synapses realization or by the preexisting pathways rearrangement. ${ }^{2}$ Neuroplasticity can be stimulated by learning new skills, illness or sensory deprivation $^{8}$ and this one may enable VI to improve other non-visual functions such as hearing, touch and smell. Thus, visual loss causes a series of functional transformations in the Central Nervous System (CNS) that leads to the individual environment adaptation. .,3,9 $^{2}$

In current systematic review, we discuss about the cross modal and multimodal neuroplasticity in VI, highlighting the possible improvements connected with them due to sensorial substitution from tactile, auditory and olfactory functions in occipital areas.

\section{Materials and Methods}

The systematic review was conducted in line with the Preferred Reporting Items for Systematic Reviews and Meta-Analyses (PRISMA statement). It also consists of a review of English language research articles about the following: Late blind, Blind, Blind congenital, Blind early, Neuroplasticity, Brain plasticity, and sensorial stimuli (e.g. auditory, tactile and olfactory stimuli). Original articles and reviews were included in this systematic review. No date or publication status restrictions were imposed. Criteria for selection were: Studies that investigated the association of neuroplasticity in visually impaired with improvement in tactile, auditory and olfactory response. Brain plasticity studies in congenital visual impairment and late visually impaired. Criteria for exclusion were: book reviews, conference proceedings, or editorials. The results were analyzed, and papers that were deemed to be relevant and of an acceptable quality were included in the analysis.

\section{Information sources}

Online searches in the database Pubmed without date or publication status restrictions were initially performed in August 2017 and repeated in October 2017 using relevant search terms: [Late Blind or Blind Congenital or Blind Early] and [Neuroplasticity or Brain plasticity] and [Auditory or tactile or olfactory]. Due to the broad nature of the review, the search was focused on the Neuroplasticity and visually impaired. Abstracts were examined for references to the research question and if the study appeared relevant, then the full text was retrieved. Reference lists of identified articles were searched for additional studies.

\section{Study selection and data extraction}

Four reviewers (PRS, TF, FC, and LS) independently read the titles and/or abstracts of the identified papers and eliminated irrelevant studies. Studies considered eligible for inclusion were read in full, and their suitability for inclusion was determined independently by them. Disagreements were managed by consensus. However, if this was not successful, consensus was sought by a fifth reviewer (VM). Data were extracted based on study design and setting. Some authors were contacted to provide supplemental information when insufficient data were provided in the study. The authors of two studies were contacted for further information having read their titles and abstracts. Two replied; since it bases the literature review fulfilled the inclusion criteria.

\section{Study selection}

Studies were included if they met the following inclusion criteria:

Initially, retrieved papers from each database were compared to remove duplicate records. Papers were then screened for eligibility based on their title and abstract, and where necessary the full-text publication was reviewed. Types of neurobiological modalities: Studies were included if they investigated brain structural connectivity using correlation among Neuroplasticity with auditory, tactile or olfactory stimulus in blind. Types of participants: Population composed of healthy individuals and individuals with visually impaired (e.g. babies, children, young adults and middle-aged). Moreover, "experimental models" using animals non-humans.

Articles were screened for eligibility based on their title and abstract, and where necessary the full-text publication was reviewed. These 197 articles were classified as meeting eligibility criteria. The next stage of the winnowing process involved full-text screening of the potentially relevant documents; of which were subsequently deemed to meet eligibility criteria. Thus, in total, 58 studies were included in the review.

\section{Results}

The neural networks reorganization in neuroplasticity in blind patients is a com- pensatory form of visual deprivation, mainly with the increased sensitivity to touch, smell and hearing (Supplementary Table 1). In an animal study, with vision deprivation before and on the fifth month, more significant activation was observed in the auditory cortex of those before. ${ }^{11,12}$

Using Magnetic Resonance Imaging (MRI), Sadato et al.,13 compared auditory cortex activity in 23 subjects; 15 blind and 8 non-blind during a tactile discrimination task. We observed that the blind activity in the post-central gyrus and the posterior parietal cortex increases with a decrease in the secondary somatosensory area. It suggests the occurrence of an association between touch and visual area. ${ }^{13}$ In another study with medium latency auditory evoked potential, a reorganization was observed at the thalamic level and in the auditory cortex in blind. ${ }^{14}$

Studies have shown that cortical thickness is related to neuroplasticity, differently between congenital and late blind. For instance, in the Jiang et al. ${ }^{15}$ study. The results demonstrated that the visual cortex of early blind are thicker than the late blind and non-blind. The researchers suggested that the lack of visual experience of early blind increases the functional synapses in the visual cortex, making it more expressive and with more synapses concerning the other study groups. Park et al.16 also analyzed the cortical thickness of 33 regions and found that the congenital visual impairment has a greater cortical thickness in areas associated with vision, while those with late blind have little or no cortical surface reduction.

\section{Discussion}

In this systematic review, we have discussed aspects about the cross modal and multimodal neuroplasticity in VI. According to the literature consulted, the occipital cortex participates in the tactile, auditory and olfactory functions improvement. Moreover, brain plasticity depends on the age of vision loss and can occur basically in two ways: cross-plasticity and multimodal. This fact corroborates to confirm what the scientists discovered more than half a decade through the neuroplasticity studies that we can highlight Paul Bach and Rita in 1967 through their observations and experiments, involving the brain-machine interface, discovered that the brain can reorganize the neural network in the absence of some sense. ${ }^{7}$

We confirm evidence for the functional improvement of other blind non-visual senses in recent studies by Ortiz-Téran et al. ${ }^{9}$ 
These authors used 11 blind after age 14 in the survey and observed through MRI a connectivity between unimodal and multimodal areas when compared to non-blind, that is, late blind and early blind when compared to the control group demonstrated improved functional connections that potentiate aspects of the sensory adaptive phenomena after visual deprivation. It may be associated with improved non-visual senses such as touch, smell, and hearing. A secondary finding in their study was the critical role of multi-sensory integration receptor areas in network reorganization and intermodal plasticity in blind individuals.

\section{The brain occipital region and its neuroplasticity for other non-visual functions}

The CNS is a constantly changing system. This characteristic called neuroplasticity consists of the tissue ability to reorganize as a normal development consequence, during the learning process or after damage to the CNS.2,3,9 Neuroplasticity is also associated with the age at which the individual became VI, i.e. if the deficiency is congenital or acquired. 17 Some evidence that the sensory modality loss can trigger a CNS reorganization, mainly at the cortical level, expanding and making more functional the information processing provided by the intact senses. Thereby, VI promotes cortical alterations that result in an improved functional capacity (Figure 1), mainly, auditory. ${ }^{10}$ These neural changes can be explained through two theories. The first is the crossplasticity that occurs in response to vision sensory deprivation in congenitally blind individuals. ${ }^{2}$ The second refers to the balance alteration in the neural networks, due to the vision absence, that enables activation of preexisting pathways, which were being inhibited by vision. ${ }^{18}$ For instance, when Ortiz-Terán et al.10 compare lateonset VI and CVI by RMF, observed that late-onset VIs showed increased multimodal integration connections in the parietal-opercular, temporoparietal junction, and ventricular pre-motor regions, whereas CVI subjects had greater associations only in the upper parietal cortex.

Therefore, neural tissue can adapt to different situations, so the vision absence does not necessarily lead to the visual cortex permanent inactivation, and it often enables the brain to modulate pre-established function areas. In healthy individuals, visual and language regions tend to "reside" in separate modules, on the other hand, we observed a fusion of these areas in CVI. ${ }^{19}$ In this way, the left frontal lobe, which is responsible for speech, can interact with the occipital lobe to assist in this func- tion control.12,13,20,22 In the study by OrtizTerán et al., 9 the local and distant connectivity integration in the cortex of late VI, CVI, and health vision individuals was compared to investigate the functional reorganization that visual deprivation provides. In this study, it was demonstrated that early and late VIs compared to individuals with health vision shows better functional connections, which potentiate the sensory adaptive process after visual deprivation. Analyzing the neuroimaging signs, the researchers concluded that the largest cortical changes occurred in the multimodal and association cortex. VIs presented better connections of multimodal integration in the parietal-opercular, temporoparietal and ventral premotor regions, whereas congenital VI showed superior connections of the parietal cortex.

\section{Anatomic-physiological changes in the visually impaired}

In congenital and early VIs, occur an increase in the cortical thickness of the occipital lobes, when compared to those with regular vision. ${ }^{15,16,22,23}$ It is due to the elimination process absence of non-functional synapses in the occipital cortex, which usually occurs in normal vision people. It leads to these synapses accumulation, culminating with a greater visual cortex thickness. ${ }^{15}$ Thus, the CNS has a selection mechanism that allows the construction and reconstruction of the synapses throughout the individual development. Therefore, not all synapses constructed are functional for the occipital cortex, vision usuful stimuli are necessary for an effective synapses selection for the correct functioning of that sense. In addition, we observed that this process occurs rapidly at early ages (between 2-4 months of life) and is maintained until the age of $11 .{ }^{24-26}$ However, this fact does not apply to late VIs, since the central nervous system is formed. $15,16,22,23$
The increase in the occipital cortex thickness, due to the accumulation of nonfunctional synapses for vision, has been correlated with better auditory performance. ${ }^{23}$ Therefore, the occipital cortex activation for non-visual tasks results in early and congenital VI better performance. Such improvement in the non-visual information processing is related to the elimination reduction process of non-functional synapses in the occipital cortex. This elimination process is a dependent experience that enables the brain to test many synapses in the occipital cortex and eliminate ineffective synapses for vision but other functions may be useful. Thus, congenital and early VIs have a greater amount of non-functional synapses for the vision recruited to auxiliary the non-visual information processing. $15,27,28$

\section{Brain plasticity differences in con- genital, early and late visual impair- ment}

The plastic changes resulting in early blind occur in a greater magnitude both from the functional and extension of the recruited brain area. ${ }^{2}$ It indicates that early VI have a greater cerebral plasticity in the occipital cortex that can reflect functional and behavioral differences when compared to late VI. ${ }^{29}$ Cross-plasticity is well established in CVI and early VI. ${ }^{30}$ An important issue is whether late VI may also benefit from compensatory changes.

Visual deprivation can cause plastic modifications that allow non-visual information to enter the occipital cortex. Two theories may be explained these neural modifications. The first would be the crossplasticity that occurs in response to vision sensory deprivation in VIs. The second one refers to the balance alteration in the neural networks, due to the vision absence, which enables the activation of preexisting pathways that were being inhibited by vision

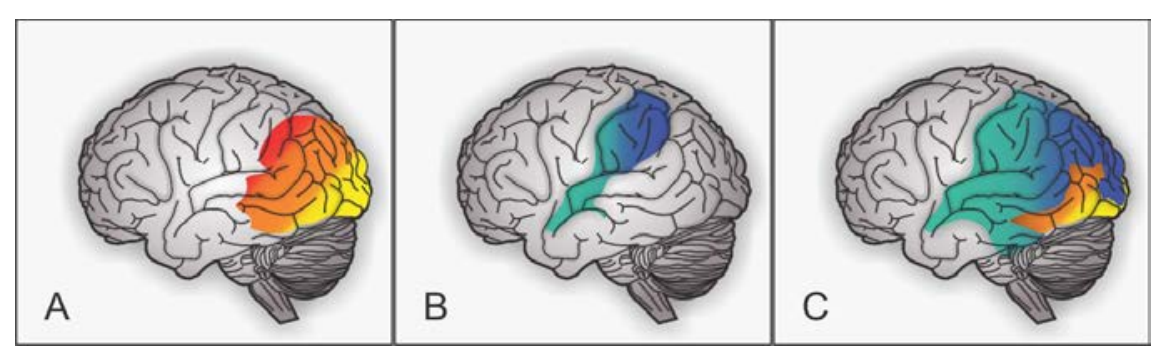

Figure 1. (A) Schematic representation of areas related to vision in healthy individuals. (B) Schematic representation of areas involved in language comprehension and semantic processes. (C) Representation of cross-neuroplasticity in congenital visually impaired patients. It is observed, an expansion of areas not related to vision to the visual cortex resulting in an improvement of non-visual senses. 
(Figure 2). ${ }^{13}$ It reinforces the idea of preexisting latent pathways between the specific sensory cortex and/or between multisensory areas and the occipital cortex. ${ }^{31}$ A neuroimaging study by Sadato et al. 13 infers that cross-plasticity is regulated for a critical period, which limits the plastic modifications in the cortex of individuals who suffered visual loss. However, other studies have shown that cross-plasticity phenomena can instead be regulated over a sensitive period, in which sensory experience has a greater influence on the behavioral and cortical development and not necessarily in a particular period (Figure 3).32,33

Kujala et al.,34 infer the possibility of rearrangement by cross-plasticity in late VI. In this study, they evaluated responses to potentials related to early and late VI events. Similar results were observed among them when subjected to the detection of sound changes. The authors concluded that cross-plasticity is related to the visual loss age. Therefore, early VI will have higher occipital cortex recruitment than those with late onset both in functionality and in recruited areas.

Evidence for the cross-plasticity existence in adults results from the investigation of the temporary visual deprivation effects in subjects with normal vision. The light deprivation in a short time increases the visual cortex excitability whereas deprivation for a brief period led to an increase in visual cortex activity. ${ }^{35}$ Pascual-Leone and Hamilton $^{36}$ developed a protocol in which non-blind volunteers had their eyes blindfolded for 5 days. The results showed an increase of the occipital cortex activity in response to tactile stimulation after 5 days of complete visual deprivation, however, when withdrawing sales, the activity was not present at the same level. These results indicate a rapid cross-plasticity in the occipital cortex after visual deprivation. In addition, Weisser et al.,37 showed that the visual deprivation in a short time is sufficient to induce cross-plasticity resulting in alterations in the occipital cortex.

Recently, Ortiz-Terán et al. 9 investigated the cortex changes of late VI individuals, CVI, and normal vision. The researchers observed that functional changes occurred in the sensory cortex, while neuroplasticity was most prominently in the multimodal and association cortex. Late deficits compared to the control group show an increase in multimodal integration connections in parietal-opercular regions, temporoparietal junction and ventricular pre-motor regions. On the other hand, the congenital deficits in comparison to the control group showed increased connections in the superior parietal and parietal opercular cortex. This study revealed the critical role of multisensory integration areas in network reorganization and cross-plasticity in VI individuals. Moreover, there was greater functional connectivity in the intermodal integration cortex in VI compared to subjects without visual impairment. These results suggest that although functional changes occur in the unimodal cortex, including the lateral occipital cortex, the large changes of neuroplasticity in VI individuals occur at the level of the multimodal integration processing regions. ${ }^{9}$ A study using positron emission tomography (PET) investigated the metabolism of glucose in the occipital cortex of individuals who lost sight during the first years of life and blindfolded individuals who had normal vision. It was observed that in the occipital cortex of VI, glucose metabolism was higher than in blindfolded individuals. ${ }^{38}$ In another study that also evaluated glucose metabolism in VI, Wanetdefalque et al.39 compared early and late visual impairment. It was found in this study that glucose metabolism is higher in early VI when compared to late VI indicat-
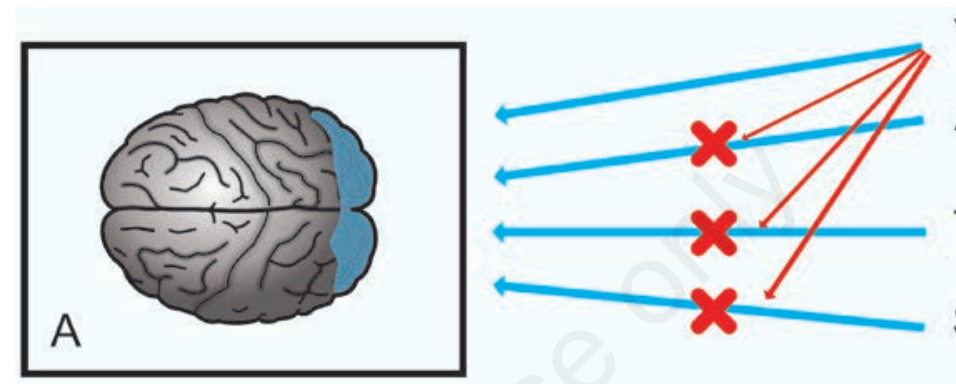

Vision

Audition

Tact

Smell
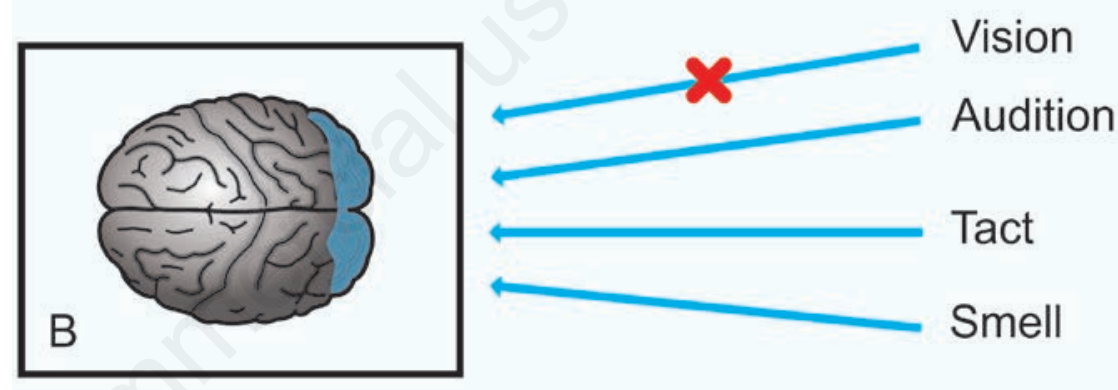

Figure 2. Schematic diagram showing multimodal neuroplasticity. (A) In people with normal vision, the occipital cortex receives and processes visual information by inhibiting preexisting pathways for non-visual functions such as hearing, touch, and smell. (B) In early visual impaired, the absence of visual information processing promotes an activation of pathways that were previously inhibited by vision resulting in improved hearing, touch, and smell.

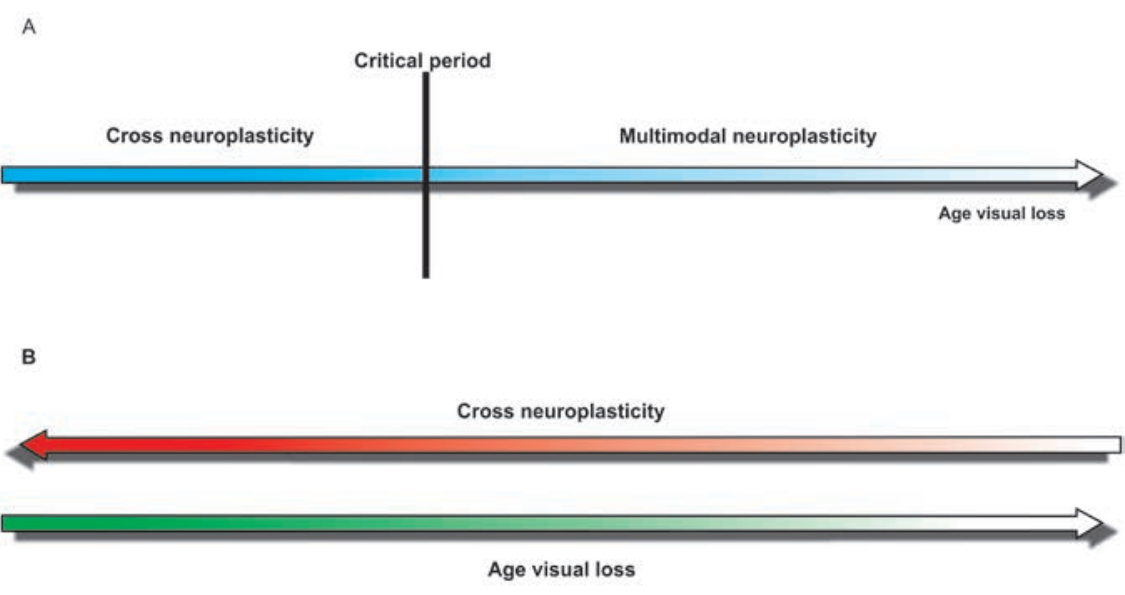

Figure 3. The illustration shows that cross-plasticity phenomena can be regulated over a sensitive period, in which sensory experience has a greater influence on behavioral and cortical development. 
ing a greater neural activity in the occipital cortex.

\section{Improvements of non-visual func- tions in visually impaired}

The cross-plasticity and multimodal theories corroborate for studies that have shown that visual cortical areas are recruited after visual loss for the processing of non-visual information. ${ }^{30}$ Cross-neuroplasticity has been associated with the visual brain areas recruitment during the sound and auditory processing. 9,12,20,21,27,32,40-44 Thus, research has reported superior performance in VI in a variety of sensory and cognitive processing tasks that include hearing, touch, and smell. 45,46 Cuevas et al. 47 investigated olfactory discrimination and odor identification abilities using olfactory identification tasks at three levels (free identification, semantic and semantic cues and phonological cues) in early VI and health vision people. The study showed that early VI significantly outperformed the control group with normal vision in odor discrimination in the free identification phase (with no semantic or phonological cues) and semantic cues. In addition, the largest difference between the groups was observed in the free identification in comparison with the semantic track and the multiple-choice conditions (semantic and phonological cues). The authors concluded that early VIs have better perceptual abilities as well as better access to information stored in semantic memory than individuals with normal vision.

The occipital cortex areas activations in VI to non-visual stimuli were observed through the sonic tasks use that resulted in the activation of those regions that would normally be associated with visual functions. Evidence lines suggest that structural and functional modifications in cross-plasticity are extremely important for VI adaptation. These evidence are based on the positive correlation between the extension in the activated visual cortex in VI and the sound location precision improvement. 23,28 This fact reinforces the idea that visual cortical areas are recruited after blindness, for the auditory information processing. 30 Although the visual loss age influences on the cross-plasticity, and the hearing functionality, ${ }^{10}$ an improved sound localization was observed after early and late visual deprivation. ${ }^{48,49}$ In addition, the functional magnetic resonance imaging use (MRI), Coullon et al. .5 compared auditory responses between individuals with congenital anophthalmia, visual impairment of early onset (in the first years of life), and in a control group with a normal vision. They found that the superior colliculus is recruited by the auditory system in congenital blindness and early onset. Auditory subcortical responses were altered as result of visual loss. Specifically, responses in the auditory thalamus were equally intense for contralateral and ipsilateral stimulation in both groups of VI, while the control presented more pronounced responses to contralateral stimulation. These findings indicate that early blindness results in a substantial reorganization of subcortical hearing responses.

Neuroplasticity evidence in adults who have passed short periods of blindfolding also reported a common transient increase in the accuracy of sound localization, i.e. there is a short period of time capable of causing changes in connections between brain regions that process information from different sensorial modalities. ${ }^{51}$ Longerterm deprivation originating from early development probably results in abnormal connectivity patterns that result in the occipital cortex recruitment for the nonvisual stimuli processing. In fact, it has been reported that in early VI there is an increase in the coupling-dependent stimuli processing between auditory and visual areas,, 52 as well as greater auditory connectivity to the visual cortex compared to individuals who have normal vision. 53

Other evidence regarding auditory improvement is related to the location of objects in space. In people with normal vision, the localization is made by a combination of vision sensory information and hearing that allows a better location estimation of an object when compared to the use of only one or other sense alone. ${ }^{54}$ Thus, the vision absence may hinder the localization of sound by VI. This difficulty can be likened to the illusion of the ventriloquist, which consists in minimizing the most visible movements of the lips and the mouth in order to confuse the observer over the sound origin, which is thus attributed to the puppet. In this situation, the observer loses the visual perception of the artist's mouth having to locate the sound only by hearing. ${ }^{54}$ In this way, vision and hearing play a fundamental role in spatial location. However, in the vision absence, VI must rely on their hearing for their location. Thus, hearing becomes more functional since it is frequently recruited to locate auditory stimuli. 55

Another functional improvement is associated with tactile perception. Bauer et $a l .56$ constructed a study model that used a tactile device composed of $32 \times 36$ pins with spacing between them of $2.4 \mathrm{~mm}$ in which each pin could be controlled independently. The control of each pin was done by software that produced symmetrical and asymmetric patterns of tactile stimulation. The authors concluded that early VI appear to exhibit enhanced abilities to detect symmetrical tactile patterns compared to the normal vision (blindfolded) control group. There is evidence that the VI occipital lobe can participate in the tactile discrimination improvement. $57-59$ Due to the importance of symmetry in visual perception, studies investigated the prior visual experience effect on forms and recognition tactile detection of tactile images, comparing VI performance and blindfolded vision. It was found that CVIs perform better in tactile perception when compared to blindfolded individuals. ${ }^{60}$ Furthermore, Goldreich and Kanics 45 compare the tactile performance of early CVI and normal subjects for vision. Participants performed a Braille reading task using the dominant finger. The results demonstrated a greater tactile acuity in late VI in relation to the group with normal vision.

In relation to the smell improvement, there are psychophysical studies that revealed differences in olfactory performance between individuals with blindness and normal vision. Cuevas et al. 47 reported that individuals with blindness have keener olfaction than those with vision in odor-free identification. In another study, BeaulieuLefebvre et al. ${ }^{61}$ demonstrated that VI subjects have a lower threshold for odor detection and are more aware of their olfactory environment. Araneda ${ }^{62}$ observed that cross-activation of occipital brain areas occurs similarly in both precocious and nonblinded blinds when olfactory stimulation occurs passively. However, when olfactory stimulation occurs actively and in the cognitive processing of some odors specific types, the brain occipital region is more stimulated in early cecum than in non-blind. In the study by Rombaux et al.,63 the results showed that early VI have a greater volume of the olfactory bulb when analyzed in MRI, in addition to superior olfactory abilities when compared to the odors discrimination and identification.

\section{Conclusions}

Vision loss is congenital, early or late, and results in CNS changes that may lead to improvements in non-visual senses, due to the expansion of non-vision related cortical areas such as hearing, touch, and smell for regions attributed to vision. In this way, the occipital cortex acts as an extension of nonvisual areas, which improves both functionality and a greater number of areas recruited in the non-visual information processing. This is due to the neuroplasticity process which is facilitated by the deficiency age. 
Thus, congenital and early VI have a higher degree of cerebral plasticity in relation to late VI. This higher degree of neuroplasticity in congenital and early VIs allows a greater integration of non-visual senses allowing these individuals a greater functionality in daily tasks such as sound location, greater tactile and olfactory distinction, that is, allows individuals a greater adaptability to the physical and social environment. In general, studies are directed to the possibility that, in the near future, science can exploit neuroplasticity in VI so that the individual with no vision can have a treatment that provides an expansion of the other senses for social readaptation. Besides, advances in the understanding of cortical plasticity may allow patients who have suffered any type of damage in brain areas to resume the activities of the damaged area by transfer to other intact brain areas.

\section{References}

1. Mariotti S, Pascolini D. Visual Impairment, Vision Loss and Blindness 2010 global estimates, and VI and blindness causes. Global Data on Visual Impairments 2010, WHO 2010.

2. Bavelier D, Neville HJ. Cross-modal plasticity: where and how? Nat Rev Neurosci 2002;3:443-42.

3. Muthusamy A, Gajendran R, Rao B. Mid-latency Auditory Evoked Potential Response Revealed as an Evidence of Neural Plasticity in Blind Individuals. Indian J Physiol Pharmacol 2014;58: 113-9.

4. Brodmann K. Vergleichende Lokalisationslehre der Grosshirnrinde. Leipzig: Johann Ambrosius Bart, 1909

5. Bach-Y-Rita P. Brain plasticity as a basis for recovery of function in humans. Neuropsychologia 1990;28:547-54.

6. Hubel D, Wiesel TN. Receptive fields and functional architecture of monkey striate cortex. J Physiol 1968;195:21543.

7. Bach-y-Rita, P. Theoretical basis for brain plasticity after a TBI. Brain Injury 2003;17:643-51.

8. Bach-Y-Rita P, Kercel SW. Sensory substitution and the human-machine interface. Trends Cogn Sci 2003;7:5416.

9. Ortiz-Terán L. Brain Plasticity in Blind Subjects Centralizes Beyond the Modal Cortices. Front Syst Neurosci 2016;10.

10. Lazzouni L, Lepore F. Compensatory plasticity: time matters. Front Hum Neurosci 2014;8:340.
11. Musacchia G, Ortiz-Mantilla S, Choudhury N, et al. Active auditory experience in infancy promotes brain plasticity in Theta and Gamma oscillations. Dev Cogn Neurosci 2017;26:9.

12. Bedny M, Richardson H, Saxe R. Visual Cortex Responds to Spoken Language in Blind Children. J Neurosci 2015;35:11674-81.

13. Sadato N, Okada T, Honda M, Yonekura Y. Critical period for crossmodal plasticity in blind humans: a functional MRI study. NeuroImage 2002;16:389-400.

14. Bavelier D, Neville HJ. Cross-modal plasticity: where and how? Nat Rev Neurosci 2002;3:443-52.

15. Jiang J, Zhu W, Shi F, et al. Thick visual cortex in the early blind. J Neurosci 2009;29:2205-11.

16. Park HJ, Lee JD, Kim EY, et al. Morphological alterations in the congenital blind based on the analysis of cortical thickness and surface area. Neuroimage 2009;47:98-106.

17. Bottari D. Sight restoration after congenital blindness does not reinstate alpha oscillatory activity in humans. Sci Rep 2016;6:24683.

18. Sadato N, Okada T, Honda M, Yonekura Y. Critical period for crossmodal plasticity in blind humans: a functional MRI study. Neuroimage 2002;16:389-400.

19. Hasson U, Andric M, Atilgana H, Collignon O. Congenital blindness is associated with large-scale reorganization of anatomical networks. NeuroImage 2016;128:362-72.

20. Arnaud L, Sato M, Ménard L, Gracco VL. Repetition suppression for speech processing in the associative occipital and parietal cortex of congenitally blind adults. PLoS One 2013;8:e64553.

21. King AJ. Crossmodal plasticity and hearing capabilities following blindness. Cell Tissue Res 2015;361:295300.

22. Bridge H, Cowey A, Ragge N, Watkins $\mathrm{K}$. Imaging studies in congenital anophthalmia reveal preservation of brain architecture in 'visual' cortex. Brain 2009; 132:3467-80.

23. Voss P, Zatorre RJ. Occipital cortical thickness predicts performance on pitch and musical tasks in blind individuals. Cereb Cortex 2011;22:2455-65.

24. Garey LJ. Structural development of the visual system of man. Hum Neurobiol 1984;3:75-80.

25. Huttenlocher PR, Dabholkar AS. Regional differences in synaptogenesis in human cerebral cortex. J Comp Neurol 1997;387:167-78.
26. Huttenlocher PR, de Courten C. The development of synapses in striate cortex of man. Hum Neurobiol 1987;6:1-9.

27. Amedi A, Raz N, Pianka P, et al. Early 'visual' córtex activation correlates with superior verbal memory performance in the blind. Nat Neurosci 2003;6:758-66.

28. Gougoux F, Zatorre RJ, Lassonde M, et al. A functional neuroimaging study of sound localization: visual córtex activity predicts performance in early-blind individuals. PLoS Biol 2005;3:e27.

29. Jiang F. Early blindness results in developmental plasticity for auditory motion processing within auditory and occipital cortex. Front Hum Neurosci 2016;10.

30. Striem-Amit E, Dakwar O, Reich L, Amedi A. The large-scale organization of "visual" streams emerges without visual experience. Cereb Cortex 2012; 22:1698-709.

31. Voss MW. Neurobiological markers of exercise-related brain plasticity in older adults. Brain Behav Immun 2013; 28:90-9.

32. Burton H, Snyder AZ, Conturo TE, et al. Adaptive changes in early and late blind: a fMRI study of Braille reading. J Neurophysiol 2002;87:589-607.

33. Voss P. A positron emission tomography study during auditory localization by late-onset blind individuals. Neuroreport 2006;17:383-8.

34. Kujala T, Alho K, Huotilainen M, et al. Electrophysiological evidence for cross-modal plasticity in humans with early- and late-onset blindness. Psychophysiology 1997;34:213-6.

35. Boroojerdi B. Reduction of human visual cortex excitability using $1-\mathrm{Hz}$ transcranial magnetic stimulation. Neurology 2000;54:152931.

36. Pascual-Leone A, Hamilton Roy. The metamodal organization of the brain. Progr Brain Res 2001;134:42745.

37. Weisser V, Stilla R, Peltier S, et al. Short-term visual deprivation alters neural processing of tactile form. Exp Brain Res 2005; 166:572-82.

38. Veraart C. Glucose utilization in human visual cortex is abnormally elevated in blindness of early onset but decreased in blindness of late onset. Brain Res 1990;510:115-21.

39. Wanet-Defalque MC, Veraart C, De Volder A, et al. High metabolic activity in the visual cortex of early blind human subjects. Brain Res 1988;446: 369-73.

40. Burton H, Diamond JB, McDermott KB. Dissociating cortical regions activated by semantic and phonological 
tasks: a FMRI study in blind and sighted people. J Neurophysiol 2003;90: 1965-82.

41. Roder B, Stock O, Bien S, et al. Speech processing activates visual cortex in congenitally blind humans. Eur J Neurosci 2002;16:930-6.

42. Bedny M, Pascual-Leone A, DodellFeder D, et al. Language processing in the occipital cortex of congenitally blind adults. Proc Natl Acad Sci USA 2011;108:4429-34.

43. Watkins KE, Cowey A, Alexander I, et al. Language networks in anophthalmia: maintained hierarchy of processing in "visual" cortex. Brain 2012;135:156677.

44. Goldreich D, Kanics IM. Tactile acuity is enhanced in blindness. J Neurosci 2003;23:3439-45.

45. Pasqualotto A, Lam JS. Congenital blindness improves semantic and episodic memory. Behav Brain Res 2013;244:162e5.

46. Kupers R, Pitto M. Compensatory plasticity and crossmodal reorganization following early visual deprivation. Neurosci Biobehav Rev 2014;41:36e52.

47. Cuevas I, Plaza P. Odour discrimination and identification are improved in early blindness. Neuropsychologia 2009;47:3079e83.

48. Voss P, Gougoux F, Lassonde M, et al.
Early- and late-onset blind individuals show supra-normal auditory abilities in far space. Curr Biol 2004;14:1734-8.

49. Fieger A, Röder B, Teder-Sälejärvi W, et al. Auditory spatial tuning in lateonset blindness in humans. J Cogn Neurosci 2006; 18:149-157.

50. Coullon GS, Jiang F, Fine I, et al. Subcortical functional reorganization due to early blindness. J Neurophysiol 2015;113:2889-99.

51. Lewald J. More accurate sound localization induced by short-term light deprivation. Neuropsychologia 2007;45:1215-22.

52. Schepers IM, Hipp JF, Schneider TR, et al. Functionally specific oscillatory activity correlates between visual and auditory cortex in the blind. Brain 2012;135:922-34.

53. Klinge C, Eippert F, Röder B, Büchel C. Corticocortical connections mediate primary visual cortex responses to auditory stimulation in the blind. J Neurosci 2010;30:12798-805

54. Alais D, Newell FN, Mamassian P. Multisensory processing in review: from physiology to behaviour. Seeing Perc 2010;23:3-38.

55. Wong M, Gnanakumaran V, Goldreich D. Tactile spatial acuity enhancement in blindness: evidence for experiencedependent mechanisms. J Neurosci
2011;31:7028-37.

56. Bauer C. Neural correlates associated with superior tactile symmetry perception in the early blind. Cortex 2015;63: 104-17.

57. Merabet L, Thut G, Murray B, et al. Feeling by sight or seeing by touch? Neuron 2004;42:173-9.

58. Poirier C, Collignon O, Scheiber C, et al. Auditory motion perception activates visual motion areas in early blind subjects. Neuroimage 2006;31:279-85.

59. Collignon O, Vandewalle G, Voss P, et al. Functional specialization for auditory-spatial processing in occipital cortex of congenitally blind humans. Proc Natl Acad Sci USA 2011;108:4435-40.

60. Bauer C, Yazzolino L, Hirsch G, et al. Neural correlates associated with superior tactile symmetry perception in the early blind. Cortex 2015;63:104-17.

61. Beaulieu-Lefebvre M. Odor perception and odor awareness in congenital blindness. Brain Res Bull 2011;84:206-9.

62. Araneda R. Cortical Plasticity and Olfactory Function in Early Blindness. Front Syst Neurosci 2016; 10.

63. Rombaux P, Potier H, Markessis E, et al. Olfactory bulb volume and depth of olfactory sulcus in patients with idiopathic olfactory loss. Eur Arch Otorhinlaryngol 2010;267:1551-6. 